\title{
Fuzzy Parameterized Dual Hesitant Fuzzy Soft Sets and Its Application in TOPSIS
}

\author{
Zahari Md Rodzi ${ }^{1,2}$, Abd Ghafur Ahmad ${ }^{1, *}$ \\ ${ }^{1}$ Faculty of Science and Technology, Universiti Kebangsaan Malaysia, Malaysia \\ ${ }^{2}$ Faculty of Computer and Mathematical Science, Universiti Teknologi MARA Kampus Seremban, Malaysia
}

Received October 30, 2019; Revised November 28, 2019; Accepted December 4, 2019

Copyright $@ 2020$ by authors, all rights reserved. Authors agree that this article remains permanently open access under the terms of the Creative Commons Attribution License 4.0 International License

\begin{abstract}
The purpose of this work is to present a new theory namely fuzzy parameterized dual hesitant fuzzy soft sets (FPDHFSSs). This theory is an extension of the existing dual hesitant fuzzy soft set whereby the set of parameters have been assigned with respective weightage accordingly. We also introduced the basic operation functions for instance intersection, union, addition and product operations of FPDHFSSs. Then, we proposed the concept of score function of FPDHFSSs of which these scores function were determined based on average mean, geometry mean and fractional score. The said scores function then were divided into the membership and non-membership elements where the distance of FPDHFSSs was introduced. The proposed distance of FPDHFSSs has been applied in TOPSIS which will be able to solve the problem of fuzzy dual hesitant fuzzy soft set environment.
\end{abstract}

Keywords Fuzzy Parameterized Dual Hesitant Fuzzy Soft Set, TOPSIS, Score Function

\section{Introduction}

Zadeh [1] presented a well-known idea namely fuzzy set. In a fuzzy set theory, it is easier to determine the membership degree of an element as compared to classical set. Since then, many extensions of fuzzy have been established. Torra [2], Vicenç Torra and Narukawa [3] presented a new ideas of fuzzy set namely hesitant fuzzy set (HFS) where the membership of target fitting to the concept is denoted by a combination of different values. Study shows that HFS is able to describe the hesitant phenomenon more comprehensively than any other extensions of fuzzy set.

Zhu et al. [4] extended the theory of HFS to dual hesitant fuzzy sets (DHFS) which has taken more information than other extensions of fuzzy sets because of the assignment of membership and non-membership values. They also believed that the more information obtained, the more efficient the decision making can be made. Of late, numerous authors have been able to establish multiple criteria decision-making (MCDM) methods and some theories under dual hesitant fuzzy environment such as [5] [17]. Then Zhang and Shu [18] presented the ideas of dual hesitant fuzzy soft sets (DHFSSs) where the integration was done based on the concept of dual hesitant fuzzy set and fuzzy soft sets. This method is then followed by Wang et al. [19].

Further, the concept of fuzzy parameterized aspect in the development of extension of fuzzy sets were introduced by Çağman et al. [20]. They introduced the fuzzy parameterized fuzzy soft set (FPFSs) and claimed that their proposed method is more efficient in handling decision making process. Bashir and Salleh [21] followed by proposing the idea of fuzzy parameterized with soft expert set by integrating an important degree for every elements in the set of parameters. Then, Geetharamani and Devi [22] introduced the model of fuzzy parameterized generalized fuzzy soft expert set as a generalization of fuzzy soft expert set by introducing a membership value for each parameter in a set of parameters with a generalized membership value. Next, Ganeshsree and Abdul Razak [23] proposed the theory of fuzzy parameterized intuitionistic fuzzy soft expert set theory and solved the problem in decision making. Al-Qudah et al. [24] presented a theory which utilizes time factor namely fuzzy parameterized complex multi-fuzzy soft expert set theory. In 2019, Al-Quran [25] introduced fuzzy parameterized complex neutrosophic soft expert sets by giving degree of importance to each parameter.

In this paper, we presented the hybrid model namely fuzzy parameterized dual hesitant fuzzy soft sets (FPDHFSSs). It is a combination of fuzzy parameterized theory and dual hesitant fuzzy soft sets (DHFSSs) by [18]. 
According to [14], DHFSSs can cope with imprecision and imperfect information than soft set and dual hesitant fuzzy set. While Zhang and Shu [18] stated that DHFSSs theory was proposed to cater for the problem of FDHFSs related to inheritance of inadequate information to the parameterization tools. This theory however can be extended to represent the multi-dimensional data set which consists the rating of each alternatives and the weightage of each parameter in a single set. In relation to that, we have assigned a weightage for each parameter in the domain of DHFSSs. All these features are included in the proposed FPDHFSSs. Subsequently, we explained every property such as union, intersection, addition and product operation function related to this theory. Then, we proposed the idea of score function for FPDHFSSs based on [26] and used it in the distance of FPDHFSSs based on the average mean, geometry mean and fractional functions to arrive at the decision-making method which will be used to solve the problem in dual hesitant fuzzy environment. A numerical example related to hiring a new employee in financial institutions is given to illustrate the feasibility of our algorithm.

This paper is prepared in the following manners: Section 2 explains the essential basic concepts for better understanding of our research work. Section 3 defines the concept of FPDHFSSs and proves some of its interesting mathematical properties. Section 4 proposes the distance measure between FPDHFSSs to study the comparison between each other's. Section 5 is dedicated for the presentation of the TOPSIS working algorithm and the application of our proposed methodology. Last but not least, Section 6 is the conclusion remarks of our theory and indicates the possibilities for future works.

\section{Preliminaries}

This section recalls some definition of hesitant fuzzy set, dual hesitant fuzzy set, fuzzy soft sets and dual hesitant fuzzy soft sets for common understanding.

Definition 1. [2], [3] Given $X$ as a fixed set, then a hesitant fuzzy set (HFS) denoted as $K$ on $X$ is a term of a function that applied to $X$ return a subset of $[0,1]$. The HFS can be written as

$$
K=\left(\left\langle x, h_{E}(x)\right\rangle \mid x \in X\right)
$$

where $h_{E}(x)$ is a set of some value in $[0,1]$, on behalf of the probable membership degree of the element $x \in X$ for the set E. For suitability, [27] $h=h_{E}(x)$ is a hesitant fuzzy element (HFE) and $H$ is the set of all HFEs.

Definition 2. [4] Given a fixed set $X$, then a dual hesitant fuzzy set (DHFS) $\Upsilon_{D}(x)$ on $X$ is a term of a function that when applied to $X$ return a subset of $[0,1]$. The DHFS can be expressed by mathematical symbol as

$$
\Upsilon_{D}(x)=(\langle x, h(x), g(x)\rangle \mid x \in X)
$$

In which $h(x)$ and $g(x)$ are two set of some values in $[0,1]$, denoting the possible membership degree and non-membership degrees for the elements $x \in X$ to the set $D$, respectively with the conditions $0 \leq \gamma, \eta \leq 1$ and $0 \leq \gamma^{+}+\eta^{+} \leq 1$ where $\gamma \in h(x)$ and $\eta \in g(x)$

$\gamma^{+} \in h^{+}(x)=\cup_{\gamma \in h(x)} \max \{y\}$ and $\eta^{+} \in g^{+}(x)=\cup \eta \in g(x) \max \{\eta\}$.

For convenience, the pair $\Upsilon_{D}(x)=\{h(x), g(x)\}$ is called dual hesitant fuzzy element (DHFE) denoted by $d=\{h, g\}$.

Definition 3. [28] Let $d=\{h, g\}, d_{1}=\left\{h_{1}, g_{2}\right\}$ and $d_{1}=\left\{h_{1}, g_{2}\right\} \quad$ be any three DHFEs with $W_{h}=W_{h_{1}}=W_{h_{2}}$ and $W_{g}=W_{g_{1}}=W_{g_{2}}$ then

$$
\begin{aligned}
& d_{1}+d_{2}=\left\{\begin{array}{c}
h^{\sigma(t)}+h_{2}^{\sigma(t)}-h_{1}^{\sigma(t)} h_{2}^{\sigma(t)} \\
1
\end{array}\right. \\
& \left.\mid t=1,2, \ldots, N_{h}\right\},\left\{g_{1}^{\sigma(s)} g_{2}^{\sigma(s)} \mid s=1,2, \ldots, N_{g}\right\}, \\
& d_{1} \times d_{2}=\left\{h_{1}^{\sigma(t)} h_{2}^{\sigma(t)} \mid t=1,2, \ldots, W_{h}\right\}, \\
& \left\{g_{1}^{\sigma(s)}+g_{2}^{\sigma(s)}-g_{1}^{\sigma(s)} g_{2}^{\sigma(s)} \mid s=1,2, \ldots, W_{g}\right\}
\end{aligned}
$$

where $W_{h}$ and $W_{g}$ represent the number of values of $h$ and $g \cdot h_{d}^{\sigma(t)}$ is the $t$ th smallest value of $h$ and $g_{d}^{\sigma(s)}$ is the $s$ th smallest value of $g$.

Definition 4. [29] Let $U$ be an initial universe set and $E$ be a universe set of parameters. A pair $(F, E)$ is called a fuzzy soft set over $U$ if $F: E \rightarrow F(U)$ where $F(U)$ is the set of all fuzzy subsets of $U$.

Definition 5. [14], [18] Let (U,E) be a soft universe and $A \subseteq E$. A pair $(\tilde{F}, A)$ is called dual hesitant fuzzy soft sets (DHFSs) over $U$ where $\tilde{F}$ is a mapping given by $\tilde{F}: A \rightarrow \operatorname{DHFS}(U)$

In general, $\tilde{F}(e)$ ) can be written as $\tilde{F}(e)=\left(\left\langle x, h_{\tilde{F}(e)}^{(x), g} \tilde{F}(e)^{(x)}\right\rangle \mid x \in X\right) \quad$ in $\quad$ which $h_{\tilde{F}(e)}(x)$ and $g_{\tilde{F}(e)}(x)$ are two sets of some values in $[0,1]$, denoting the possible membership degree and non-membership degrees that object $x$ holds on parameter $e$, respectively. 


\section{Fuzzy Parameterized Dual Hesitant Fuzzy Soft Sets}

This section explains the combinations of weightage for each parameter and DHFSs by introducing a new hybrid model called FPDHFSSs.

Definition 6. Let $U$ be a universe set, $E$ be the set of parameters, and $X$ be the set of alternatives. Then $\Psi_{K}$ is called fuzzy parameterized dual hesitant fuzzy soft sets (FPDHFSSs) over $U$ denoted by

$$
\begin{aligned}
& \Psi_{K}=\left\{\left(\frac{e_{i}}{\mu_{K}\left(e_{i}\right)}, \frac{x_{j}}{\Upsilon_{K}\left(x_{j}\right)}\right)\right. \\
& \left.\mid e_{i} \in E, x_{j} \in X, \Upsilon_{K}\left(x_{j}\right) \in D H F S(U)\right\}
\end{aligned}
$$

where $\mu_{K}\left(e_{i}\right)$ is a value of fuzzy parameterized set between 0 to 1 , while $\Upsilon_{K}\left(x_{j}\right)=\left\{h_{K}^{v p}\left(x_{j}\right), g_{K}^{v}{ }_{K}\left(x_{j}\right)\right\}$ is represented in dual hesitant fuzzy set. Consider the set of all fuzzy parameterized dual hesitant fuzzy soft sets on $U$ denoted by $\operatorname{FPDHFSS}(U)$. The value of $h_{K}^{v p}\left(x_{j}\right)$ represent the smallest of $p$-th value of $h$ and $g_{K}^{v_{q}}\left(x_{j}\right)$ represent the smallest of $q$-th value of $g$. The number of values of $h_{K}^{v}{ }_{K}\left(x_{j}\right)$ and $g_{K}^{v p}\left(x_{j}\right)$ are denoted by $W_{h_{K}\left(x_{j}\right)}$ and $W_{g_{K}\left(x_{j}\right)}$ respectively. In this paper we use the concept of pessimistic by adding the maximum values in it until it has the same length.

Example 1. Let $K$ consists of four shortlisted alternatives denoted by $X=\left\{h_{1}, h_{2}, h_{3}, h_{4}\right\}$ and three parameters namely $E=\left\{e_{1}, e_{2}, e_{3}\right\}$. Suppose that $\left\{\frac{e_{1}}{0.4}, \frac{e_{2}}{0.8}, \frac{e_{3}}{0.2}\right\}$ and the rating of alternatives according to parameters are given as

$$
\begin{gathered}
\Upsilon\left(e_{1}\right)=\left\{\frac{h_{1}}{(\{0.2,0.3\},\{0.4,0.6,\}}, \frac{h_{2}}{(\{0.5,0.6\},\{0.1,0.2\}}\right. \\
\left., \frac{h_{3}}{(\{0.4,0.5\},\{0.3\})}\right\} \\
\Upsilon\left(e_{2}\right)=\left\{\frac{h_{1}}{(\{0.4,0.5,0.6\},\{0.2,0.4\}}, \frac{h_{2}}{(\{0.7,0.8,0.9\},\{0.1\}},\right. \\
\left.\frac{h_{3}}{(\{0.4,0.5,0.6\},\{0.2,0.4\})}\right\}
\end{gathered}
$$

$\Upsilon\left(e_{3}\right)=\left\{\frac{h_{1}}{(\{0.1,0.2,0.4\},\{0.3,0.6\}}, \frac{h_{2}}{(\{0.6,0.7\},\{0.2,0.3\}}\right.$,

$\left.\frac{h_{3}}{(\{0.3,0.5\},\{0.4\})}\right\}$

Then the FPDHFSSs is given by

$\Psi_{K}=\left\{\left(\frac{e_{1}}{0.4}, \frac{h_{1}}{(\{0.2,0.3,0.3\},\{0.4,0.6\})}, \frac{h_{2}}{(\{0.5,0.6,0.6\},\{0.1,0.2\})}\right.\right.$,

$\frac{h_{3}}{(\{0.4,0.5,0.5\},\{0.3,0.3\})}\left(\frac{e_{2}}{0.5}, \frac{h_{1}}{(\{0.4,0.5,0.6\},\{0.2,0.4,\})}\right.$,

$\left.\frac{h_{2}}{(\{0.7,0.8,0.9\},\{0.1,0.1\})}, \frac{h_{3}}{(\{0.4,0.5,0.6\},\{0.2,0.4\})}\right)$

$\left(\frac{e_{3}}{0.7} \frac{h_{1}}{(\{0.1,0.2,0.4\},\{0.2,0.6\})}, \frac{h_{2}}{(\{0.6,0.7,0.7\},\{0.2,0.3\})}\right.$,

$\left.\left.\frac{h_{3}}{(\{0.1,0.3,0.5\},\{0.4,0.4\})}\right\}\right)$.

Definition 7. Let $\Psi_{K}$ be FPDHFSS over $U$. Then $\Psi_{K}=\left\{\left(\frac{e_{i}}{0}, \frac{x_{j}}{\{\tilde{0}\},\{\tilde{1}\}}\right): e_{i} \in E, x_{j} \in X\right\} \quad$ is called an empty FPDHFSS set and denoted by $\Psi_{\phi}$.

Definition 8. Let $\Psi_{K}$ be FPDHFSS over $U$. Then $\Psi_{K}=\left\{\left(\frac{e_{i}}{1}, \frac{x_{j}}{\{\tilde{1}\},\{\tilde{0}\}}\right): e_{i} \in E, x_{j} \in X\right\} \quad$ is called a universal FPDHFSS and denoted by $\Psi_{\tau}$.

Proposition 1. Let $\Psi_{K}, \Psi_{L}$ and $\Psi_{M}$ be any three FPDHFSSs. Then the following results hold:

i) $\Psi_{K} \subseteq \Psi_{\tau}$,

ii) $\Psi_{\phi} \subseteq \Psi_{\tau}$,

iii) $\Psi_{K} \subseteq \Psi_{K}$,

iv) $\Psi_{K} \subseteq \Psi_{L}$ and $\Psi_{L} \subseteq \Psi_{M}$ then $\Psi_{K} \subseteq \Psi_{M}$,

v) $\Psi_{K}=\Psi_{L}$ and $\Psi_{L}=\Psi_{M}$ then $\Psi_{K}=\Psi_{M}$,

vi) $\Psi_{K} \subseteq \Psi_{L}$ and $\Psi_{L} \subseteq \Psi_{K}$ then $\Psi_{K}=\Psi_{L}$.

Proof: The proof is straightforward.

Definition 9. Let $\Psi_{K}$ be FPDHSS. Then the complement of $\Psi_{K}=\left\{\left(\frac{e_{i}}{\mu_{K}\left(e_{i}\right)}, \frac{x_{j}}{\Upsilon_{K}\left(x_{j}\right)}\right)\right.$ is $\left.\mid e_{i} \in E, x_{j} \in X, \Upsilon_{K}\left(x_{j}\right) \in \operatorname{DHFS}(U)\right\}$

denoted by $\Psi_{K}^{c}=\left\{\left(\frac{e_{i}}{\mu_{K}\left(e_{i}\right)}, \frac{x_{j}}{\Upsilon_{K}\left(x_{j}\right)}\right)\right.$ $\left.\mid e_{i} \in E, x_{j} \in X, \Upsilon_{K}\left(x_{j}\right) \in \operatorname{DHFS}(U)\right\}^{c}$ 
defined by

$$
\begin{aligned}
& \Psi_{K}^{c}=\left\{\left(\frac{e_{i}}{\left(1-\mu_{K}\left(e_{i}\right)\right)},\left(\Upsilon_{K}\left(x_{j}\right)\right)^{c}\right)\right. \\
& \left.\mid e_{i} \in E, x_{j} \in X, \Upsilon_{K}\left(x_{j}\right) \in \operatorname{DHFS}(U)\right\} .
\end{aligned}
$$

where

$$
\left(\Upsilon_{K}\left(x_{j}\right)\right)^{c}=\left(\left\{g_{K}^{v q}\left(x_{j}\right)\right\},\left\{h_{K}^{v p}\left(x_{j}\right)\right\}\right) .
$$

Example 2. Reconsider Example 1. The complement of $\Psi_{K}$ is given as

$$
\begin{aligned}
& \Psi_{K}^{c}=\left\{\left(\frac{e_{1}}{0.6}, \frac{h_{1}}{(\{0.4,0.6\},\{0.2,0.3,0.3\})}, \frac{h_{2}}{(\{0.1,0.2\},\{0.5,0.6,0.6\})},\right.\right. \\
& \left.\frac{h_{3}}{(\{0.3,0.3\},\{0.4,0.5,0.5\})}\right),\left(\frac{e_{2}}{0.5}, \frac{h_{1}}{(\{0.2,0.4,\},\{0.4,0.5,0.6\})},\right. \\
& \left.\frac{h_{2}}{(\{0.1,0.1\},\{0.7,0.8,0.9\})}, \frac{h_{3}}{(\{0.2,0.4\},\{0.4,0.5,0.6\})}\right) \\
& \left(\frac{e_{3}}{0.3}, \frac{h_{1}}{(\{0.2,0.6\},\{0.1,0.2,0.4\})}, \frac{h_{2}}{(\{0.2,0.3\},\{0.6,0.7,0.7\})},\right. \\
& \left.\left.\frac{h_{3}}{(\{0.4,0.4\},\{0.1,0.3,0.5\})}\right\}\right) .
\end{aligned}
$$

Proposition 2 Let $\Psi_{K}$ be FPDHSS(U). Then the following results hold:

$$
\begin{aligned}
& \text { i) }\left(\Psi_{K}^{C}\right)^{C}=\Psi_{K} \\
& \text { ii) } \Psi_{\phi}^{C}=\Psi_{\tau}
\end{aligned}
$$

Proof. The proofs are straightforward.

Definition 10. Let $\Psi_{K}$ and $\Psi_{L}$ be FPDHFSSs over $U$. Supposed that $W_{h_{K}\left(x_{j}\right)}=W_{h_{L}\left(x_{j}\right)}$ and $W_{g_{K}}\left(x_{j}\right)=W_{g_{L}\left(x_{j}\right)}$. Then the OR operations of $\Psi_{K}$ and $\Psi_{L}$, denoted by $\Psi_{K} \breve{\cap} \Psi_{L}$ is defined by $\Psi_{K} \frown \Psi_{L}=\left\{\left(\frac{e_{i}}{\min \left(\mu_{K}\left(e_{i}\right), \mu_{L}\left(e_{i}\right)\right)}, \Upsilon_{K}\left(x_{j}\right) \curvearrowright \Upsilon_{L}\left(x_{j}\right)\right)\right.$ $\left.e_{i} \in E, x_{j} \in X, \Upsilon_{K}\left(x_{j}\right), \Upsilon_{L}\left(x_{j}\right) \in \operatorname{DHFS}(U)\right\}$ where

$$
\begin{aligned}
& \Upsilon_{K}\left(x_{j}\right) \curvearrowright \Upsilon_{\sim}\left(x_{j}\right)=\left\{h_{K}^{v p}\left(x_{j}\right) \cup h_{L}^{v} p_{\left(x_{j}\right)}\right\} \\
& \left\{g_{K}^{v q_{K}}\left(x_{j}\right) \cap g_{L}^{v q}\left(x_{j}\right)\right\} .
\end{aligned}
$$

Example 3. Let $\Psi_{K}$ is given in Example 1 and $\Psi_{L}$ is given below

$$
\begin{aligned}
& \Psi_{L}=\left\{\left(\frac{e_{1}}{0.7}, \frac{h_{1}}{(\{0.4,0.5,0.6\},\{0.2,0.3\}}, \frac{h_{2}}{(\{0.3,0.4,0.5\},\{0.3,0.5\}},\right.\right. \\
& \left.\frac{h_{3}}{(\{0.4,0.6,0.6\},\{0.3,0.4\})}\right),\left(\frac{e_{2}}{0.4}, \frac{h_{1}}{(\{0.2,0.3,0.6\},\{0.3,0.4\}},\right.
\end{aligned}
$$

$\left.\frac{h_{2}}{(\{0.5,0.5,0.5\},\{0.2,0.4\}}, \frac{h_{3}}{(\{0.3,0.5,0.6\},\{0.3,0.4\})}\right)$

$\left(\frac{e_{3}}{0.2}, \frac{h_{1}}{(\{0.4,0.6,0.7\},\{0.2,0.3\}}, \frac{h_{2}}{(\{0.6,0.7,0.8\},\{0.1,0.1\}}\right.$

,$\left.\left.\frac{h_{3}}{(\{0.2,0.3,0.5\},\{0.5,0.5\})}\right)\right\}$.

The OR operation of $\Psi_{K}$ and $\Psi_{L}$ is given as

$\Psi_{K} \check{\frown} \Psi_{L}=\left\{\left(\frac{e_{1}}{0.4}, \frac{h_{1}}{(\{0.2,0.3,0.3\},\{0.4,0.6\}}\right.\right.$,

$\left.\frac{h_{2}}{(\{0.3,0.4,0.5\},\{0.3,0.5\}}, \frac{h_{3}}{(\{0.4,0.5,0.5\},\{0.3,0.3\})}\right)$,

$\left(\frac{e_{2}}{0.4}, \frac{h_{1}}{(\{0.3,0.4,0.6\},\{0.3,0.4\}}, \frac{h_{2}}{(\{0.5,0.5,0.5\},\{0.2,0.4\}}\right.$,

$\left.\frac{h_{3}}{(\{0.3,0.5,0.6\},\{0.3,0.4\})}\right),\left(\frac{e_{3}}{0.2}, \frac{h_{1}}{(\{0.1,0.2,0.4\},\{0.2,0.6\}}\right.$,

$\left.\left.\frac{h_{2}}{(\{0.6,0.7,0.7\},\{0.2,0.3\}}, \frac{h_{3}}{(\{0.1,0.3,0.5\},\{0.4,0.4\})}\right)\right\}$.

Proposition 3. Let $\Psi_{K}, \zeta_{L}$ and $\zeta_{M}$ be any three FPDHFSSs. Then the following results hold:
i) $\Psi_{K} \tilde{\cap} \Psi_{K}=\Psi_{K}$
ii) $\Psi_{\phi} \tilde{\cap} \Psi_{K}=\Psi_{\phi}$
iii) $\Psi_{K} \tilde{\cap} \Psi_{\tau}=\Psi_{K}$
iv) $\Psi_{K} \tilde{\cap} \Psi_{L}=\Psi_{L} \tilde{\cap} \Psi_{K}$

Proof. The proofs are straightforward.

Definition 11. Let $\Psi_{K}$ and $\Psi_{L}$ be FPDHFSSs over

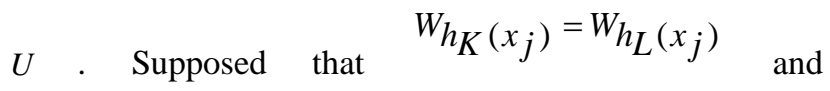
$W_{g_{K}\left(x_{j}\right)}=W_{g_{L}\left(x_{j}\right)}$. Then the intersection of $\Psi_{K}$ and $\Psi_{L}$, denoted by $\Psi_{K} \cup \Psi_{L}$ is defined by,

$$
\begin{aligned}
& \Psi_{K} \breve{\cup} \Psi_{L}=\left\{\left(\frac{e_{i}}{\min \left(\mu_{K}\left(e_{i}\right), \mu_{L}\left(e_{i}\right)\right)}, \frac{x_{j}}{\Upsilon_{K}\left(x_{j}\right) \cup \Upsilon_{L}\left(x_{j}\right)}\right)\right. \\
& \left.\mid e_{i} \in E, x_{j} \in X, \Upsilon_{K}\left(x_{j}\right), \Upsilon_{L}\left(x_{j}\right) \in \operatorname{DHFS}(U)\right\}
\end{aligned}
$$


where

$$
\begin{aligned}
& \Upsilon_{K}\left(x_{j}\right) \cup \Upsilon_{L}\left(x_{j}\right)=\left\{h_{K}^{v p}\left(x_{j}\right) \cap h_{L}^{v} p_{\left(x_{j}\right)}\right\} \\
& ,\left\{g_{K}^{v_{q}}\left(x_{j}\right) \cup g_{L}^{v^{q}}\left(x_{j}\right)\right\}
\end{aligned}
$$

Example 4. Let $\Psi_{K}$ and $\Psi_{L}$ are given in Example 3. The intersections of $\Psi_{K}$ and $\Psi_{L}$ is given as

$$
\begin{aligned}
& \Psi_{K} \tilde{\cup} \Psi_{L}=\left\{\left(\frac{e_{1}}{0.7}, \frac{h_{1}}{(\{0.4,0.5,0.6\},\{0.2,0.3\}},\right.\right. \\
& \left.\frac{h_{2}}{(\{0.5,0.6,0.6\},\{0.1,0.2\}}, \frac{h_{3}}{(\{0.4,0.6,0.6\},\{0.3,0.3\})}\right), \\
& \left(\frac{e_{2}}{0.5}, \frac{h_{1}}{(\{0.4,0.5,0.6\},\{0.2,0.4\}}, \frac{h_{2}}{(\{0.7,0.8,0.9\},\{0.1,0.1\}},\right. \\
& \left.\frac{h_{3}}{(\{0.4,0.5,0.6\},\{0.2,0.4\})}\right),\left(\frac{e_{3}}{0.7} \frac{h_{1}}{(\{0.5,0.6,0.7\},\{0.2,0.3\}},\right. \\
& \left.\left.\frac{h_{2}}{(\{0.6,0.7,0.8\},\{0.1,0.1\}}, \frac{h_{3}}{(\{0.2,0.3,0.5\},\{0.3,0.4\})}\right)\right\}
\end{aligned}
$$

Proposition 4. Let $\Psi_{K}$ and $\Psi_{L}$ be any three FPDHFSSs. Then the following results hold:
i) $\Psi_{K} \tilde{\cup} \Psi_{K}=\Psi_{K}$
ii) $\Psi_{\phi} \tilde{\cup} \Psi_{K}=\Psi_{K}$
iii) $\Psi_{K} \tilde{\cup} \Psi_{\tau}=\Psi_{\tau}$
iv) $\Psi_{K} \tilde{\cup} \Psi_{L}=\Psi_{L} \tilde{\cup} \Psi_{K}$

Proof. The proofs are straightforward.

Definition 12. Let $\Psi_{K}$ and $\Psi_{L}$ be FPDHFSSs over $U$. Supposed that $W_{h_{K}\left(x_{j}\right)}=W_{h_{L}}\left(x_{j}\right)$ and $W_{g_{K}\left(x_{j}\right)}=W_{g_{L}\left(x_{j}\right)}$. Then, the union of $\Psi_{K}$ and $\Psi_{M}$, denoted by $\Psi_{K} \oplus \Psi_{L}$ is defined by,

$$
\begin{aligned}
& \Psi_{K} \oplus \Psi_{L}=\left\{\left(\frac{e_{i}}{\left(\mu_{K}\left(e_{i}\right) \oplus \mu_{L}\left(e_{i}\right)\right)}, \frac{x_{j}}{\Upsilon_{K}\left(x_{j}\right) \oplus \Upsilon_{L}\left(x_{j}\right)}\right)\right. \\
& \left.\mid e_{i} \in E, x_{j} \in X, \Upsilon_{K}\left(x_{j}\right), \Upsilon_{L}\left(x_{j}\right) \in \operatorname{DHFS}(U)\right\}
\end{aligned}
$$

where,

$$
\begin{aligned}
& \Upsilon_{K}\left(x_{j}\right) \oplus \Upsilon_{L}\left(x_{j}\right)=\left\{h_{K}^{v p}\left(x_{j}\right)+h_{L}^{v p}\left(x_{j}\right)-\right. \\
& \left.h_{K}^{v p}\left(x_{j}\right) \cdot h_{L}^{v p}\left(x_{j}\right)\right\},\left\{g_{K}^{v q}\left(x_{j}\right) \cdot g_{L}^{v q}\left(x_{j}\right)\right\}
\end{aligned}
$$

Definition 13. Let $\Psi_{K}$ and $\Psi_{L}$ be FPDHFSSs over U. Supposed that $W_{h_{K}\left(x_{j}\right)}=W_{h_{L}}\left(x_{j}\right)$ and $W_{g_{K}\left(x_{j}\right)}=W_{g_{L}\left(x_{j}\right)}$. Then, the union of $\Psi_{K}$ and
$\Psi_{M}$, denoted by $\Psi_{K} \otimes \Psi_{L}$ is defined by,

$$
\begin{aligned}
& \Psi_{K} \otimes \Psi_{L}=\left\{\left(\frac{e_{i}}{\left(\mu_{K}\left(e_{i}\right) \otimes \mu_{L}\left(e_{j}\right)\right)}, \Upsilon_{K}\left(x_{j}\right) \otimes \Upsilon_{L}\left(x_{j}\right)\right)\right. \\
& \left.\mid e_{i} \in E, x_{j} \in X, \Upsilon_{K}\left(x_{j}\right), \Upsilon_{L}\left(x_{j}\right) \in \operatorname{DHFS}(U)\right\}
\end{aligned}
$$

where,

$$
\begin{aligned}
& \Upsilon_{K}\left(x_{j}\right) \otimes \Upsilon_{L}\left(x_{j}\right)=\left\{h_{K}^{v p}\left(x_{j}\right) \cdot h_{L}^{v_{p}}\left(x_{j}\right)\right\}, \\
& \left\{g_{K}^{v q}\left(x_{j}\right)+g_{L}^{v q}\left(x_{j}\right)-g_{K}^{v q}\left(x_{j}\right) \cdot g_{L}^{v q}\left(x_{j}\right)\right\}
\end{aligned}
$$

Example 5. Reconsider example 1 and example 3. The addition and product operations of $\Psi_{K}$ and $\Psi_{L}$ are as follows:

$$
\begin{aligned}
& \Psi_{K} \oplus \Psi_{L}=\left\{\left(\frac{e_{1}}{0.82}, \frac{h_{1}}{(\{0.52,0.65,0.72\},\{0.08,0.18\}},\right.\right. \\
& \left.\frac{h_{2}}{(\{0.65,0.76,0.8\},\{0.03,0.1\}}, \frac{h_{3}}{(\{0.64,0.8,0.8\},\{0.09,0.09\})}\right), \\
& \left(\frac{e_{2}}{0.7}, \frac{h_{1}}{(\{0.58,0.7,0.84\},\{0.06,0.16\}}, \frac{h_{2}}{(\{0.85,0.9,0.95\},\{0.02,0.04\}}\right. \\
& \left., \frac{h_{3}}{(\{0.58,0.75,0.84\},\{0.06,0.16\})}\right), \\
& \left(\frac{e_{3}}{0.76}, \frac{h_{1}}{(\{0.55,0.68,0.82\},\{0.04,0.18\}},\right. \\
& \left.\left.\frac{h_{2}}{(\{0.81,0.91,0.94\},\{0.02,0.03\}}, \frac{h_{3}}{(\{0.28,0.51,0.75\},\{0.12,0.16\})}\right)\right\} \\
& \Psi_{K} \otimes \Psi_{L}=\left\{\left(\frac{e_{1}}{0.28}, \frac{h_{2}}{(\{0.08,0.15,0.18\},\{0.52,0.72\}},\right.\right. \\
& \frac{h_{3}}{(\{0.15,0.24,0.3\},\{0.37,0.6\})}, \frac{h_{1}}{(\{0.16,0.3,0.3\},\{0.51,0.51\})} \\
& \left(\frac{e_{2}}{0.2}, \frac{h_{1}}{(\{0.12,0.2,0.36\},\{0.44,0.64\}}, \frac{h_{3}}{(\{0.35,0.4,0.45\},\{0.28,0.46\}}\right. \\
& \left., \frac{h_{3}}{(\{0.12,0.25,0.36\},\{0.44,0.64\})}\right),\left(\frac{e_{3}}{0.14},\right. \\
& \frac{h_{1}}{(\{0.05,0.12,0.28\},\{0.36,0.72\}}, \frac{h_{3}}{(\{0.36,0.49,0.56\},\{0.28,0.37\}}, \\
& \left.\left.\frac{h_{3}}{(\{0.02,0.09,0.25\},\{0.58,0.64\})}\right)\right\}
\end{aligned}
$$

Proposition 4. Let $\Psi_{K}$ and $\Psi_{L}$ be any two FPDHFSSs. Then the following results hold:
i) $\quad \Psi_{K} \oplus \Psi_{L}=\Psi_{L} \oplus \Psi_{K}$
ii) $\Psi_{K} \otimes \Psi_{L}=\Psi_{L} \otimes \Psi_{K}$
iii) $\left(\Psi_{K} \oplus \Psi_{L}\right)^{C}=\Psi_{K}^{C} \otimes \Psi_{L}^{C}$
iv) $\left(\Psi_{K} \otimes \Psi_{L}\right)^{C}=\Psi_{K}^{C} \oplus \Psi_{L}^{C}$ 
Proof. The proof i and ii are straightforward.

According to definition 10 and 3, for all e we have

$$
\begin{aligned}
& \left(\Psi_{K} \oplus \Psi_{L}\right)^{c}=\left(\frac{e_{i}}{\left.\left(1-\mu_{K}\left(e_{i}\right) \oplus \mu_{L}\left(e_{i}\right)\right)\right)}\right. \\
& \left\{g_{K}^{v_{q}}\left(x_{j}\right) \cdot g_{L}^{v_{q}}\left(x_{j}\right)\right\}, \\
& \left.\left\{h_{K}^{v p}\left(x_{j}\right)+h_{L}^{v} p_{\left(x_{j}\right)}\right\}: e_{i} \in E, x_{j} \in X\right) \\
& =\left(\frac{e_{i}}{\left(1-\mu_{K}\left(e_{i}\right)\right)},\left\{g_{K}^{v_{q}}\left(x_{j}\right)\right\},\left\{h_{K}^{v_{p}}\left(x_{j}\right)\right\}\right. \\
& \left.: e_{i} \in E, x_{j} \in X\right) \otimes \\
& \left(\frac{e_{i}}{\left(1-\mu_{L}\left(e_{i}\right)\right)},\left\{g_{L}^{v_{q}}\left(x_{j}\right)\right\},\left\{h_{L}^{v_{p}}\left(x_{j}\right)\right\}:\right. \\
& \left.e_{i} \in E, x_{j} \in X\right) \\
& =\Psi_{K}^{C} \otimes \Psi_{L}^{c}
\end{aligned}
$$

The result can be proved in a similar way.

\section{The Score Function and Distance of Fuzzy Parameterized Dual Hesitant Fuzzy Soft Sets}

The score function of FPDHFSSs and the distance between two FPDHFSSs play an important tool in this research. A lot of distance measures have been proposed for hesitant fuzzy sets and dual hesitant fuzzy sets. First, let's take a look at the distance property as follows:

Definition 14. Let $\Psi_{K}$ and $\Psi_{L}$ be FPDHFSSs over $U$. Distance measure between $\Psi_{K}$ and $\Psi_{L}$ is defined as $d\left(\Psi_{K}, \Psi_{L}\right)$ which satisfies the following properties:

(P1) $0 \leq d\left(\Psi_{K}, \Psi_{L}\right) \leq 1$ (Boundary)

(P2) $d\left(\Psi_{K}, \Psi_{L}\right)=d\left(\Psi_{L}, \Psi_{K}\right)$ (Symmetry)

(P3) $d\left(\Psi_{K}, \Psi_{L}\right)=0$ if only if $\Psi_{K}=\Psi_{K}$.

(Reflexivity)

Definition 15. Let $\Psi_{K}$ be FPDHFSSs over $U$. The following mathematical functions can be considered as the score functions of FPDHFSSs;

1. The arithmetic means score function of FPDHFSSs.

$$
\begin{aligned}
& S_{A M}\left(\Psi_{K}\right)=\left(s_{A M}^{h}, S_{A M}^{g}\right)= \\
& \mu_{K}\left(e_{i}\right) \frac{1}{n} \sum_{1=i}^{n} h_{K}^{v p}\left(x_{j}\right), \\
& \left.\mu_{K}\left(e_{i}\right) \frac{1}{m} \sum_{1=j}^{m} g_{K}^{v p}\left(x_{j}\right)\right)
\end{aligned}
$$

2. The geometric-mean score function of FPDHFSSs.

$$
\begin{aligned}
& S_{G M}\left(\Psi_{K}\right)=\left(s_{G M}^{h}, S_{G M}^{g}\right)= \\
& \mu_{K}\left(e_{i}\right)\left(\prod_{1=i}^{p} h_{K}^{v_{p}}\left(x_{j}\right)\right)^{\frac{1}{p}}, \\
& \mu_{K}\left(e_{i}\right)\left(\prod_{1=j}^{q} g_{K}^{v_{p}}\left(x_{j}\right)\right)^{\frac{1}{q}}
\end{aligned}
$$

3. and the fractional score function of FPDHFSSs.

$$
\begin{aligned}
& S_{F}\left(\Psi_{K}\right)=\left(s_{F}^{h}, s_{F}^{g}\right)= \\
& \mu_{K}\left(e_{i}\right)\left(\frac{\prod_{1=i}^{n} h_{K}^{v p}\left(x_{j}\right)}{\prod_{1=i}^{n} h_{K}^{v^{\prime} p}\left(x_{j}\right)+\prod_{1=i}^{n} 1-h_{K}^{v^{v}}\left(x_{j}\right)}\right), \\
& \mu_{K}\left(e_{i}\right)\left(\frac{\prod_{1=j}^{m} g_{K}^{v_{q}}\left(x_{j}\right)}{\prod_{1=j}^{m} g_{K}^{v_{q}}\left(x_{j}\right)+\prod_{1=i}^{n}{ }^{1-g_{K}{ }^{v}{ }_{K}\left(x_{j}\right)}}\right)
\end{aligned}
$$

Definition 16. Let $\Psi_{K}$ and $\Psi_{L}$ be FPDHFSSs over $U$. The distance measure between $\Psi_{K}$ and $\Psi_{L}$ is given by these mathematical formulae

1. the FPHDFSSs average mean distances

$$
\begin{aligned}
& d_{A M}\left(\Psi_{K}, \Psi_{K}\right)=\left(\frac{1}{n} \sum\left|S_{A M}^{h}(K)-S_{A M}^{h}(L)\right|^{\lambda}\right) \\
& \left.+\left|S_{A M}^{g}(K)-S_{A M}^{g}(L)\right|^{\lambda}\right)^{\frac{1}{\lambda}}
\end{aligned}
$$


2. the FPHDFSSs geometry mean distances

$$
\begin{aligned}
& d_{G M}\left(\Psi_{K}, \Psi_{K}\right)=\left(\frac{1}{n} \sum\left|s_{G M}^{h_{\breve{F}}}(\alpha)-\mu_{\breve{G}} \cdot S_{G M}^{h_{G}}(\beta)\right|^{\lambda}\right. \\
& \left.+\left|s_{G M}^{g_{\breve{F}}}(\alpha)-s_{G M}^{g_{\breve{G}}}(\beta)\right|^{\lambda}\right)^{\frac{1}{\lambda}}
\end{aligned}
$$

3. the FPDHFSS fractional distances

$$
\begin{aligned}
& d_{F}\left(\Psi_{K}, \Psi_{K}\right)=\left(\frac{1}{n} \sum\left|S_{F r}^{h_{\breve{F}}}(\alpha)-S_{F r}^{h_{\breve{G}}}(\beta)\right|^{\lambda}\right. \\
& \left.+\left|S_{F r}^{g_{\breve{F}}}(\alpha)-S_{F r}^{g_{\breve{G}}}(\beta)\right|^{\lambda}\right)^{\frac{1}{\lambda}}
\end{aligned}
$$

when $\lambda=1$, it is also known as Hamming distance of FPDHFSSs and when $\lambda=2$, is it also known as Euclidean distance of FPDHFSSs.

Example 6. Let $\Psi_{K}$ and $\Psi_{L}$ be FPDHFSSs over $U$

$$
\begin{aligned}
& \Psi_{K}=\left\{\left(\frac{e_{1}}{0.5}, \frac{h_{1}}{(\{0.2,0.3,0.3\},\{0.4,0.6\})},\right.\right. \\
& \left.\frac{h_{2}}{(\{0.5,0.6,0.6\},\{0.1,0.2\})}, \frac{h_{3}}{(\{0.4,0.5,0.5\},\{0.3,0.3\})}\right) \\
& \left(\frac{e_{2}}{0.6}, \frac{h_{1}}{(\{0.3,0.5,0.6\},\{0.2,0.4,\})},\right. \\
& \left.\left.\frac{h_{2}}{(\{0.5,0.6,0.7\},\{0.2,0.3\})}, \frac{h_{3}}{(\{0.6,0.7,0.8\},\{0.1,0.2\})}\right)\right\}
\end{aligned}
$$

and

$$
\begin{aligned}
& \Psi_{L}=\left\{\left(\frac{e_{1}}{0.3}, \frac{h_{1}}{(\{0.2,0.4,0.5\},\{0.4,0.5\}},\right.\right. \\
& \left.\frac{h_{2}}{(\{0.7,0.8,0.8\},\{0.1,0.2\}}, \frac{h_{3}}{(\{0.3,0.4,0.6\},\{0.3,0.4\})}\right), \\
& \left(\frac{e_{2}}{0.8}, \frac{h_{1}}{(\{0.4,0.5,0.6\},\{0.2,0.3\}}, \frac{h_{2}}{(\{0.4,0.5,0.6\},\{0.4,0.4\}}\right. \\
& \left.\left., \frac{h_{3}}{(\{0.6,0.7,0.7\},\{0.2,0.3\})}\right)\right\}
\end{aligned}
$$

The FPHDFSSs average mean distances measure between $\Psi_{K}$ and $\Psi_{L}$ is given as

$$
\begin{aligned}
& d_{A M}\left(\Psi_{K}, \Psi_{L}\right)= \\
& \frac{1}{n} \sum\left(\left|0.5 \frac{(0.2+0.3+0.3)}{3}-0.3 \frac{(0.2+0.5+0.5)}{3}\right|+\right. \\
& \left|0.5 \frac{(0.4+0.6)}{2}-0.3 \frac{(0.4+0.5)}{2}\right| \\
& +\left|0.5 \frac{(0.5+0.6+0.6)}{3}-0.3 \frac{(0.7+0.8+0.8)}{3}\right|
\end{aligned}
$$

$$
\begin{aligned}
& +\left|0.5 \frac{(0.1+0.2)}{2}-0.3 \frac{(0.1+0.2)}{2}\right| \\
& +\left|0.5 \frac{(0.4+0.5+0.5)}{3}-0.3 \frac{(0.3+0.4+0.6)}{3}\right|+ \\
& \left|0.5 \frac{(0.3+0.3)}{2}-0.3 \frac{(0.3+0.4)}{2}\right| \\
& +\left|0.6 \frac{(0.3+0.5+0.6)}{3}-0.8 \frac{(0.4+0.5+0.6)}{3}\right|+ \\
& +\left|0.6 \frac{(0.2+0.4)}{2}-0.8 \frac{(0.2+0.3)}{2}\right|+ \\
& +\left|0.6 \frac{(0.2+0.3)}{2}-0.8 \frac{(0.4+0.4)}{2}\right| \\
& +\left|0.6 \frac{(0.6+0.7+0.8)}{3}-0.8 \frac{(0.6+0.7+0.7)}{3}\right| \\
& \left.+\left|0.6 \frac{(0.1+0.2)}{2}-0.8 \frac{(0.2+0.3)}{2}\right|\right) \\
& =0.1572
\end{aligned}
$$

\section{The Applications of FPDHFSSs Distance Measures' in TOPSIS}

In this section, we utilize the proposed distance measure to develop TOPSIS method for multiple attribute group decision making with fuzzy parameterized dual hesitant fuzzy information. TOPSIS is a multiple criteria method to identify solutions from a finite set of alternatives, which was initially proposed by [30]. The logic of TOPSIS [30] is to define the positive ideal solution and negative ideal solution. The best result should have the shortest distance after the positive ideal solution and farthest after the negative ideal solution.

The algorithm of the distance of FPDHFSSs in TOPSIS is

Step 1. Set up the decision matrix R.

Step 2. Create the score function of the decision matrix based on Definition 15.

Step 3. Determine the positive and negative ideal solutions.

Step 4. Compute the distance of alternative to the positive ideal solution and negative ideal solution as in Definition 15.

Step 5. Determine the relative closeness to the ideal solution $C_{i}=\frac{d^{-}}{d^{-}+d^{+}}$.

Step 6. Rank each alternative based on the relative closeness. 
Example 7. Consider a financial institution that is looking for a candidate to fill in the position for Vice President of Product \& Development department. There are four potential candidates who are namely $\left(h_{1}, h_{2}, h_{3}, h_{4}\right)$ to be selected. The three attributes considered in selecting the best candidate are qualification $\left(e_{1}\right)$, working experience $\left(e_{2}\right)$, and good team-player $\left(e_{3}\right)$. Suppose the institution evaluates the optimal four candidates under various attributes with FPDHFSSs. The overall FPDHFSSs is given by:

$$
\begin{aligned}
& \Psi_{A}=\left\{\left(\frac{e_{1}}{0.35}, \frac{h_{1}}{(\{0.3,0.4,0.5\},\{0.2,0.3,0.4\})},\right.\right. \\
& \frac{h_{2}}{(\{0.4\},\{0.4,0.5,0.6\})}, \frac{h_{3}}{(\{0.1,0.2,0.5\},\{0.4,0.5\})}, \\
& \left.\frac{h_{4}}{(\{0.5,0.6,0.8\},\{0.2\})}\right),\left(\frac{e_{2}}{0.45}, \frac{h_{1}}{(\{0.2,0.3,0.6\},\{0.1,0.3\})},\right. \\
& \left(\frac{h_{2}}{(\{0.7,0.8,0.9\},\{0.1\})}, \frac{h_{3}}{(\{0.6,0.7,0.8\},\{0.1,0.2\})},\right. \\
& \left.\frac{h_{4}}{(\{0.4,0.6,0.7\},\{0.1,0.2,0.3\})}\right),\left(\frac{e_{3}}{0.15}, \frac{h_{1}}{(\{0.3,0.4,0.6\},\{0.2,0.3\})},\right. \\
& \frac{h_{2}}{(\{0.2,0.4,0.5\},\{0.2,0.3,0.4\})}, \frac{h_{3}}{(\{0.3,0.4,0.5\},\{0.2,0.3,0.4\})}, \\
& \left.\left.\frac{h_{4}}{(0.6,0.7\},\{0.1,0.2\})}\right)\right\} .
\end{aligned}
$$

Step 1. The decision matrix of FPDHFSSs, $\mathrm{R}$ is obtained as in Table 1.

Table 1. Decision matrix of FPDHFSSs

\begin{tabular}{|c|c|c|c|}
\hline & $e_{1}(0.35)$ & $e_{2}(0.45)$ & $e_{3}(0.15)$ \\
\hline$h_{1}$ & $\{0.3,0.4,0.5)$, & $\{0.2,0.3,0.6)$, & $\{0.3,0.4,0.6)$, \\
& $\{0.2,0.3,0.4\}$ & $\{0.1,0.3,0.3\}$ & $\{0.2,0.3,0.3\}$ \\
\hline$h_{2}$ & $\{0.4,0.4,0.4)$, & $\{0.7,0.8,0.9)$, & $\{0.2,0.4,0.5)$, \\
& $\{0.4,0.5,0.6\}$ & $\{0.1,0.1,0.1\}$ & $\{0.2,0.3,0.4\}$ \\
\hline \multirow{2}{*}{$h_{3}$} & $\{0.1,0.2,0.5)$, & $\{0.6,0.7,0.8)$, & $\{0.3,0.4,0.5)$, \\
\hline \multirow{2}{*}{$h_{4}$} & $\{0.4,0.5,0.5\}$ & $\{0.1,0.2,0.2\}$ & $\{0.2,0.3,0.4\}$ \\
\hline
\end{tabular}

Step 2. In this application, we show the calculations of the geometry mean score function of FPDHFSSs. The decision matrix for this calculations is portrayed in Table 2.

Table 2. Score function of decision matrix of FPDHFSSs

\begin{tabular}{|l|c|c|c|}
\hline & $e_{1}$ & $e_{2}$ & $e_{3}$ \\
\hline$h_{1}$ & $(0.1370,0.1010)$ & $(0.1486,0.0936)$ & $(0.0624,0.0393)$ \\
\hline$h_{2}$ & $(0.1400,0.1726)$ & $(0.3581,0.0450)$ & $(0.0433,0.0740)$ \\
\hline$h_{3}$ & $(0.0754,0.1625)$ & $(0.3128,0.0714)$ & $(0.0323,0.0696)$ \\
\hline$h_{4}$ & $(0.2175,0.0700)$ & $(0.2483,0.0818)$ & $(0.0997,0.0238)$ \\
\hline
\end{tabular}

Step 3. The positive and negative ideal solutions are given in Table 3.

Table 3. Positive and negative ideal solutions

\begin{tabular}{|c|c|c|c|}
\hline & $e_{1}$ & $e_{2}$ & $e_{3}$ \\
\hline$A^{+}$ & $\{0.2175,0.0700\}$ & $\{0.3581,0.0450\}$ & $\{0.0997,0.0238\}$ \\
\hline$A^{-}$ & $\{0.0754,0.1726\}$ & $\{0.1486,0.0936\}$ & $\{0.0323,0.0740\}$ \\
\hline
\end{tabular}

Step 4. Using Definition 16 for $\lambda=1$, we have the distance of alternative to the positive ideal solution and negative ideal solutions shown in Table 4.

Table 4. Distance from positive and negative ideal solutions

\begin{tabular}{|l|l|l|}
\hline & $d_{G M}^{+}$ & $d_{\overline{G M}}^{-}$ \\
\hline$h_{1}$ & 0.2352 & 0.1051 \\
\hline$h_{2}$ & 0.1492 & 0.2248 \\
\hline$h_{3}$ & 0.1953 & 0.1661 \\
\hline$h_{4}$ & 0.1158 & 0.2188 \\
\hline
\end{tabular}

Step 5. The relative closeness for $\lambda=1,2,4,6,8,10$ to the ideal solutions is shown in Table 5 .

Table 5. Correlation coefficient based on the geometry mean distance of FPDHFSSs

\begin{tabular}{|c|c|c|c|c|c|c|}
\hline$\lambda$ & 1 & 2 & 4 & 6 & 8 & 10 \\
\hline$h_{1}$ & 0.3192 & 0.3088 & 0.2775 & 0.2661 & 0.2612 & 0.2587 \\
\hline$h_{2}$ & 0.5378 & 0.6012 & 0.6501 & 0.6637 & 0.6681 & 0.6699 \\
\hline$h_{3}$ & 0.3239 & 0.4597 & 0.5222 & 0.5326 & 0.5351 & 0.5358 \\
\hline$h_{4}$ & 0.7638 & 0.6539 & 0.5913 & 0.5740 & 0.5680 & 0.5657 \\
\hline
\end{tabular}

Step 6. Thus, we have the ranking of each alternative where $h_{4} \succ h_{2} \succ h_{1} \succ h_{3}$.

The relative closeness for geometry mean score function, average mean score function and fractional score function are given in Figure 1, Figure 2 and Figure 3 respectively.

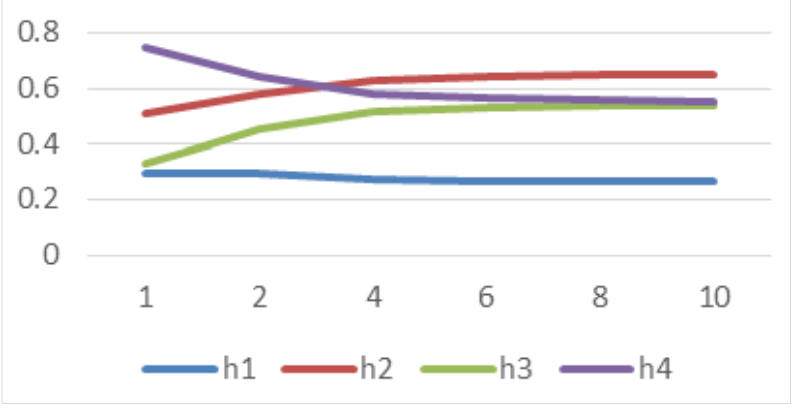

Figure 1. Relative Closeness based on average mean score function of FPDHFSSs 


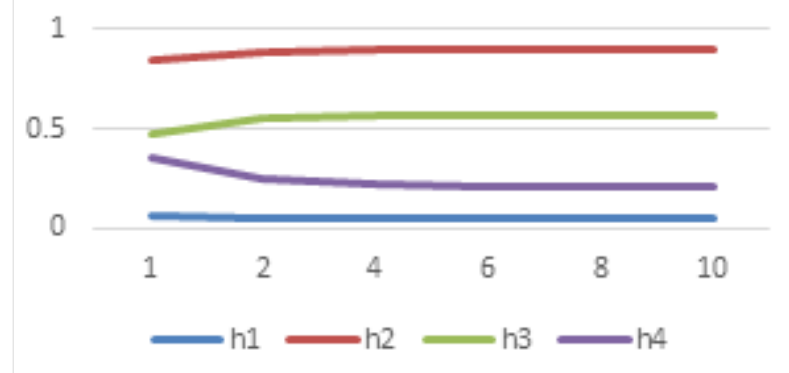

Figure 2. Relative Closeness based on fractional score function of FPDHFSSs

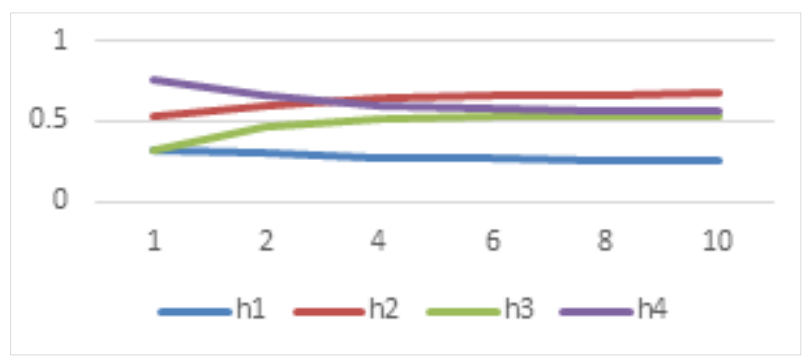

Figure 3. Relative Closeness based on geometry mean score function of FPDHFSSs

Figures 1, 2 and 3 show that the ranking of alternatives can change based on the value of $\lambda$. The ranking changes for average mean score function and geometry mean score function while for the fraction score function the ranking of alternatives remain the same for $\lambda=1,2,4,6,8,10$.

\section{Conclusions}

We presented the fuzzy parameterized dual hesitant fuzzy soft set by giving an important degree to each parameter in combination of dual hesitant fuzzy set and fuzzy soft set. In addition, we also discussed AND and OR operations and some other properties related to the functions by giving illustrative examples together with some basic operations of FPDHFSSs like equality, subset, complement, intersection and union. The advantage of using FPDHFSSs is in representing information of two dimensions for one object simultaneously or objects with multi-dimensional characterization properties in one model. Then we also introduced three distance measures based on average mean, geometry mean and fractional of dual hesitant fuzzy set to be applied onto TOPSIS to solve the problems in dual hesitant fuzzy soft set environment. The novelty of FPDHFSSs seems to be in its capability to offer a concise and inclusive representation of two-dimensional hesitant information as well as the suitable parameterization and the thoughts of the decision makers, all in a single set. In this regard, we are eager to widen our study to other multi-criteria decision-making models and applications for real life uncertainty and vagueness problems.

\section{REFERENCES}

[1] L. Zadeh, “Zadeh_FuzzySetTheory_1965.pdf,” Information and Control, vol. 8. pp. 338-353, 1965.

[2] V. Torra, “Hesitant Fuzzy Sets,” Int. J. Intell. Syst., vol. 25, pp. 529-539, 2010.

[3] V. Torra and Y. Narukawa, "On hesitant fuzzy sets and decision,” IEEE Int. Conf. Fuzzy Syst., pp. 1378-1382, 2009.

[4] B. Zhu, Z. Xu, and M. Xia, "Dual hesitant fuzzy sets," J. Appl. Math., vol. 2012, 2012.

[5] Z. Wu, F. Zhang, J. Sun, W. Wang, and X. Tang, "Novel parameterized utility function on dual hesitant fuzzy rough sets and its application in pattern recognition,” Inf., vol. 10, no. 2, 2019.

[6] X. Tang, S. Yang, and W. Pedrycz, "Multiple attribute decision-making approach based on dual hesitant fuzzy Frank aggregation operators,” Appl. Soft Comput. J., vol. 68, pp. 525-547, 2018.

[7] S. Narayanamoorthy, L. Ramya, D. Baleanu, J. V. Kureethara, and V. Annapoorani, "Application of normal wiggly dual hesitant fuzzy sets to site selection for hydrogen underground storage,” Int. J. Hydrogen Energy, no. xxxx, 2019.

[8] José Carlos R. Alcantud and J. Z. Gustavo Santos-García, Xindong Peng, "Dual Extended Hesitant Fuzzy Sets," Symmetry (Basel)., vol. 11, no. 714, pp. 1-13, 2019.

[9] D. Liang, M. Wang, Z. Xu, and D. Liu, "Risk appetite dual hesitant fuzzy three-way decisions with TODIM,” Inf. Sci. (Ny)., vol. 507, pp. 585-605, 2020.

[10] J. Zhang, G. Hegde, J. Shang, and X. Qi, "Evaluating Emergency Response Solutions for Sustainable Community Development by Using Fuzzy Multi-Criteria Group Decision Making Approaches: IVDHF-TOPSIS and IVDHF-VIKOR,” Sustainability, vol. 8, no. 4, p. 291, 2016.

[11] Z. Ren, Z. Xu, and H. Wang, "Dual hesitant fuzzy VIKOR method for multi-criteria group decision making based on fuzzy measure and new comparison method,” Inf. Sci. (Ny)., vol. 388-389, pp. 1-16, 2017.

[12] Z. Su, Z. Xu, H. Liu, and S. Liu, "Distance and similarity measures for dual hesitant fuzzy sets and their applications in pattern recognition,” J. Intell. Fuzzy Syst., vol. 29, no. 2, pp. 731-745, 2015.

[13] P. Singh, “A new method for solving dual hesitant fuzzy assignment problems with restrictions based on similarity measure,” Appl. Soft Comput. J., vol. 24, pp. 559-571, 2014.

[14] Yan-ping He, “An Approach to Dual Hesitant Fuzzy Soft Set Based on Decision Making,” Adv. Intell. Syst. Comput., vol. 367, 2016.

[15] G. Maity, D. Mardanya, S. K. Roy, and G. W. Weber, “A new approach for solving dual-hesitant fuzzy transportation problem with restrictions,” Sadhana - Acad. Proc. Eng. Sci., 
vol. 44, no. 4, pp. 1-11, 2019.

[16] D. Liang, A. P. Darko, Z. Xu, and M. Wang, “Aggregation of dual hesitant fuzzy heterogenous related information with extended Bonferroni mean and its application to MULTIMOORA,” Comput. Ind. Eng., vol. 135, no. September 2018, pp. 156-176, 2019.

[17] J. Li, J. qiang Wang, and J. hua Hu, “Consensus building for hesitant fuzzy preference relations with multiplicative consistency,” Comput. Ind. Eng., vol. 128, no. December 2018, pp. 387-400, 2019.

[18] H. Zhang and L. Shu, "dual hesitant fuzzy soft set and its properties,” Adv. Intell. Syst. Comput., vol. 367, pp. 171182, 2016.

[19] H. Garg and R. Arora, "Dual Hesitant Fuzzy Soft Aggregation Operators and Their Application in Decision-Making," Cognit. Comput., vol. 10, no. 5, pp. 769-789, 2018.

[20] N. Çağman, F. Çıtak, and S. Enginoğlu, "Fuzzy Parameterized Fuzzy Soft Set Theory and Its Applications," Turkish J. Fuzzy Syst., vol. 1, no. 1, pp. 21-35, 2010.

[21] M. Bashir and A. R. Salleh, "Fuzzy Parameterized Soft Expert Set,” Abstr. Appl. Anal., vol. 2012, pp. 1-15, 2012.

[22] G. Geetharamani and S. Devi, "Fuzzy Parameterized generalised Fuzzy Soft Expert Set,” Abstr. Appl. Anal., vol. 9, no. 10, pp. 239-246, 2015.

[23] S. Ganeshsree and S. Abdul Razak, "Fuzzy Parameterized Intuitionistic Fuzzy Soft Expert Set Theory and its Application in Decision Making,” Int. J. Soft Comput., vol. 11, no. 2, pp. 52-63, 2016.

[24] Y. Al-Qudah, M. Hassan, and N. Hassan, "Fuzzy Parameterized Complex Multi-Fuzzy Soft Expert Set Theory and Its Application in Decision-Making," Symmetry (Basel)., vol. 11, no. 3, p. 358, 2019.

[25] A. Al-Quran, N. Hassan, and S. Alkhazaleh, "Fuzzy Parameterized Complex Neutrosophic Soft Expert Set for Decision under Uncertainty,” Symmetry (Basel)., vol. 11, no. 3, p. 382, 2019.

[26] B. Farhadinia, “A series of score functions for hesitant fuzzy sets,” Inf. Sci. (Ny)., vol. 277, pp. 102-110, 2014.

[27] M. Xia and Z. Xu, "Hesitant fuzzy information aggregation in decision making,” Int. J. Approx. Reason., vol. 52, no. 3, pp. 395-407, 2011.

[28] R. Wang, “Generalized Dual Hesitant Fuzzy Bonferroni Mean with Its Application to Supplier Selection,” in IEEE International Conference on Logistics, Informatics and Service Sciences, 2017, no. July, pp. 998-1003.

[29] A. Kharal and B. Ahmad, “On fuzzy soft sets,” Adv. Fuzzy Syst., vol. 2009, 2009.

[30] C.-L. Hwang and K. Yoon, Multiple Attribute Decision Making — Methods and Applications. 1981. 